\title{
El análisis de flujos energéticos como herramienta de análisis territorial; el caso de la
} Región Metropolitana de Barcelona

Energy flow analysis as a tool for territorial analysis; the case of the Barcelona Metropolitan Region

María Christina Fragkou*, Teresa Vicent**, Xavier Gabarrell**

\section{Filiación}

* Departamento de Geografía, Universidad de Chile

E mail: mariac.fragkou@gmail.com

** Instituto de Ciencias y Tecnología Ambiental, Universidad Autónoma de Barcelona

E mail: teresa.vicent@uab.cat,xavier.gabarrell@uab.cat

Primera versión recibida en: 11 de septiembre, 2015

Última versión recibida en: 21 de diciembre, 2015

\section{Resumen}

La diversificación de las fuentes energéticas usadas a lo largo de la historia, resultado del creciente consumo de los sistemas socioeconómicos, ha sido un factor clave tanto en determinar nuestra relación con el medio ambiente, como en la evolución de la economía global y el ordenamiento territorial a todas las escalas geográficas. Una gran mayoría de los estudios relacionados se ha enfocado en el análisis del consumo energético urbano y de los factores que lo condicionan. Este artículo presenta un marco metodológico basado en el concepto de metabolismo energético para el análisis de sistemas urbano-regionales. La metodología se aplica a un sistema costero del Mediterráneo. Los resultados demuestran que el estudio de los flujos energéticos es una herramienta valiosa para la interpretación de la estructura económica de un sistema y la evaluación de su autosuficiencia energética. Adicionalmente, es capaz de revelar posibles patrones de consumo energético asociados a distintos tipos de economías y de urbanización.

\section{Palabras clave}

Consumo energético; metabolismo socioeconómico; densidad urbana; Barcelona

\begin{abstract}
The diversification of energy resources throughout human history, as a result of the increasing energy demands of socioeconomic systems, has been a decisive factor in shaping our relationship with the environment, the structure of global economy and land use planning at all geographical scales. An important part of related studies has focused on the study of urban energy consumption and the factors that affect this. This paper presents a methodological framework based on the concept of energy metabolism for the analysis of urban-regional systems. The methodology is applied on an urban coastal Mediterranean system. The results reveal that energy flow analysis is a useful tool for the interpretation of the economic structure of a system and the assessment of its self-sufficiency in energy generation. What is more, it is apt for revealing possible consumption patterns associated to different types of economy and urbanisation.
\end{abstract}

\section{Keywords}

Energy consumption; socioeconomic metabolism; urban density; Barcelona 


\section{Sumario}

Introducción

1 Balances y metabolismo de energía en los estudios de sustentabilidad

2 El caso de estudio

3 Marco metodológico

4 Resultados y Discusión

4.1 Cálculo del mix eléctrico del sistema

4.2 Análisis de la evolución del consumo final de energía por fuente y sector

4.3 Generación local de energía y evaluación de la auto-suficiencia del sistema

4.4 Análisis del consumo energético a nivel municipal

5 Conclusiones

Referencias

\section{Introducción}

El problema energético que afrontamos hoy en día no se podría analizar sin considerar el contexto social, técnico y económico actual. Tal como lo expresó Kastoriadis hace más de treinta años, la cuestión de la energía es simplemente un problema de la sociedad y de cómo esta es estructurada y políticamente organizada (Kastoriadis \& Cohn-Bendit, 1981). Las transiciones energéticas que han sucedido durante la historia de la humanidad, han formado e influenciado todos los aspectos de organización social y las interrelaciones socio-naturales, resultando en dramáticos cambios en el metabolismo socio-económico y la colonización de los sistemas naturales (Fischer-Kowalski \& Haberl, 1998). El uso del petróleo como una fuente energética eficiente, barata y abundante ha implicado fundamentales cambios, principalmente en los modos de producción y consumo; ha desplazado la mano de obra humana desde la agricultura a la industria y más adelante al sector de servicios, facilitando la concentración de población y la inevitable creación de grandes centros urbanos.

El consiguiente transporte de bienes y personas más rápido y barato también afectó la organización espacial y los patrones metabólicos tanto a nivel global como local. Los sistemas socio-económicos ya no se restringen por los usos de suelo y tampoco se limitan en su medio ambiente limítrofe para la extracción de recursos naturales y materias primas para sus economías; el transporte barato en una economía global permite la importación de materiales y mercancías del lugar del mundo que tenga menos costos de producción (Sempere, 2007). En el ámbito urbano, el transporte barato tuvo como consecuencia la extensión descontrolada de las metrópolis modernas y la facilitación de su segregación en áreas de usos de suelo especializados, implicando la dependencia automovilística.

Si bien la consecuente expansión urbana se ha estudiado extensivamente como un fenómeno socio-económico, tanto a nivel internacional como en Chile, la pluralidad de los estudios relevantes se enfocan en sus consecuencias sociales (Ortiz y Morales, 2002) y ambientales (Romero y Vásquez, 2005), mientras los aspectos energéticos de este fenómeno urbano tienden de ignorarse por la gran mayoría de la bibliografía. Sin embargo, la infraestructura y la forma, y en consecuencia la planificación, urbana afectan dramáticamente el consumo de energía en las ciudades, principalmente en el sector doméstico y de transporte. Este hecho vincula estrechamente la planificación urbana con la eficiencia energética y sustentabilidad de la urbes, y su rol tanto en la contribución como en la mitigación del cambio climático. 
Es por todo lo anterior que el presente trabajo analiza el uso del metabolismo energético como una herramienta para el análisis de sistemas urbano-regionales. Se investiga la utilidad del concepto y de los indicadores derivados para el análisis energético sectorial y temporal de los sistemas estudiados. Para servir a este objetivo, se desarrolla una metodología para una contabilización simple de los flujos energéticos de un sistema urbano-regional. La herramienta metodológica se aplica a dos distintas escalas geográficas en un área urbana del Mediterráneo como caso de estudio.

En las siguientes secciones se da inicialmente una revisión de los principales métodos de análisis energético; a continuación se presenta el caso de estudio y el marco metodológico usado, seguido por un capítulo con los resultados y la discusión de estos. Finalmente, los principales hallazgos de este trabajo se resumen en la sección de conclusiones.

\section{Balances y metabolismo de energía en los estudios de sustentabilidad}

El concepto del metabolismo energético de un sistema socioeconómico se basa en el del metabolismo social, lo cual fue propuesto por investigadores con una visión biofísica hacía la economía. Ellos han detectado analogías entre el metabolismo humano y la función de los sistemas socioeconómicos en términos de consumo material y energético (Haberl et al., 2004). En las disciplinas de Economía Ecológica y Ecología Industrial, los sistemas socioeconómicos se reconocen como sistemas abiertos que son incorporados en su medio ambiente natural y que dependen de él mediante un constante intercambio de materiales y energía, como se ilustra en la Fig. 1. Las modernas economías industriales se ven como organismos vivos que ingieren materias primas y recursos energéticos (flujos de entrada), los cuales se metabolizan para generar bienes y servicios, y excretan residuos y emisiones (flujos de salida) (Matthews et al., 2000). El concepto del metabolismo energético se usa para describir y cuantificar este intercambio de energía entre una economía y el medio ambiente.

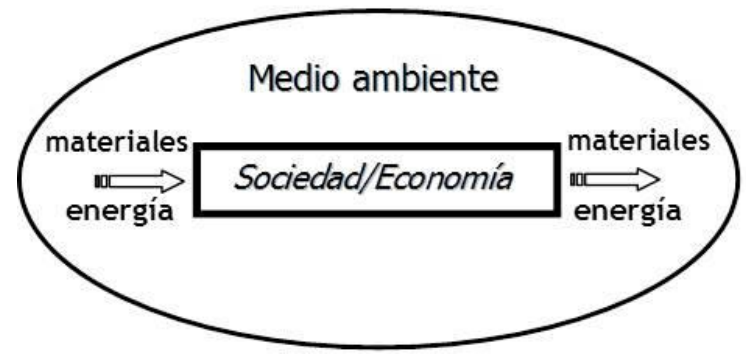

Fig. 1 El sistema economía/sociedad

Fuente: Eurostat, 2001

Los problemas ecológicos que están asociados al metabolismo energético son centrales en la agenda del desarrollo sustentable. La identificación de los flujos energéticos de un sistema es crucial en el análisis de la eficiencia y el progreso de la estructura y el crecimiento económico, de la organización social y de la innovación tecnológica, entre otras variables de la sustentabilidad (Krausmann et al., 2004). Unas revisiones exhaustivas sobre el origen y los precursores del metabolismo energético se han presentado por Martinez-Alier $(1987 ; 2004)$ y Fischer-Kowalski (1998). Suh (2005) también ha realizado una revisión de la evolución del análisis de los flujos energéticos en el campo de la ecología, analizando cada una de las metodologías usadas.

La importancia de los flujos energéticos ha resultado en su inclusión en las cuentas estadísticas nacionales (Agencia Internacional de Energía, 1995; Naciones Unidas, 1997) y la derivación de 
numerosos indicadores de producción y consumo de energía (vea por ejemplo Agencia Europea de Medio Ambiente, 1999; Eurostat, 1999; Naciones Unidas - Departamento de Asuntos Económicos y Sociales, 2001; Organismo Internacional de Energía Atómica, 2005). Sin embargo, los balances y estadísticas energéticos convencionales1 solo consideran la energía usada para la producción de calor, electricidad y luz (Krausmann \& Harbel, 2002), omitiendo la fracción de biomasa que no se utiliza como combustible para la generación de calor o electricidad, usando los valores caloríficos netos de los materiales.

La metodología de Análisis de Flujos de Energía (Energy Flow Accounting - EFA) complementa estos métodos considerando todas las entradas energéticas directas en el sistema (Haberl et al., 2004). Más concretamente, contabiliza todas las entradas de biomasa en el sistema, incluyendo la fracción que se usa para la nutrición humana y de animales, la cual es significante incluso en el metabolismo de sistemas industriales (Haberl, 2006). Los indicadores que se pueden derivar incluyen las Entradas Directas de Energía y la Extracción Doméstica de Energía. Además, las cuentas de EFA se calculan a partir del valor calorífico bruto de todos los materiales considerados (Krausmann \& Harbel, 2002).

Otras metodologías usadas para calcular los flujos energéticos de un sistema, que frecuentemente son complementarias, incluyen enfoques de multi-criterio y multi-escalares (Ramos-Martín et al., 2007a), análisis "input-output" para flujos energéticos (Thi Anh Tuyet \& Ishihara, 2006), análisis extendido de exergía (Sciubba, 2003), análisis de flujos de exergía (Talens et al., 2008), análisis de emergía (Ulgiati et al., 1994) y Análisis de Ciclo de Vida (Gasol et al., 2009).

El estudio del metabolismo energético a varias escalas geográficas y temporales ha sido el tema de numerosos trabajos, usando una amplia gama de herramientas metodológicas. Algunos ejemplos son, entre otros, el estudio de Haberl (2006) sobre el metabolismo energético de la humanidad durante el último millón de años, contabilizando también el consumo de biomasa2, usando el Análisis de Flujos de Energía, y el trabajo de Krausmann \& Haberl (2002) sobre la transición de la economía austríaca a su etapa industrial, desde una perspectiva de flujos energéticos. Adicionalmente, varios estudios se han realizado con un enfoque de escala local o regional (por ejemplo, Sundkvist et al., 1999; Oliver-Solà et al., 2007), incluyendo un informe sobre el metabolismo energético de Cataluña (Ramos-Martín et al., 2007b).

Por lo que se refiere a los sistemas urbanos, Brugmann (1996) menciona que la planificación energética era el primer elemento en desarrollarse en temas de gestión urbana, cuando "(...) ya en la década de los 1980 los planificadores de energía urbana aplicaban balances de materiales y energía para determinar las reservas, flujos y las eficiencias de conversión de las fuentes energéticas urbana (...)." Desde entonces se han presentado varios trabajos sobre la interrelación del medio ambiente urbano con su entorno natural y los factores que afectan el consumo energético en los centros urbanos (Huang, 1998; Huang et al., 2007). En su estudio del metabolismo de ocho regiones metropolitanas de todo el mundo, Kennedy et al. (2007) mencionan las condiciones climáticas, el costo de la energía, la edad de la ciudad y el estado de su desarrollo, como factores clave que afectan el consumo energético y el perfil metabólico de una ciudad.

Un mayor número de estudios se ha enfocado en la relación entre la densidad urbana y el consumo energético. Mindali et al. (2004) estudiaron la interrelación entre el consumo

\footnotetext{
${ }^{1}$ Para una distinción entre los balances energéticos y las estadísticas energéticas vea Haberl (2001)

2 Analizando su evolución mediante las transiciones desde las sociedades de recolectores-depredadores, a las agrícolas y hasta las industriales.
} 
energético en los sistemas de transporte y la densidad urbana, basadas en el trabajo pionero de Newman \& Kenworthy (1989) que sugirió una relación negativa entre los dos factores; los autores argumentan que esta correlación no se puede dar por hecha en todos los casos. Larivière \& Lafrance (1999) demuestran mediante un modelo que las ciudades de alta densidad urbana usan menos electricidad por habitante que las de baja densidad. Aún así, los autores notan que la influencia de este factor en el consumo de gasolina es mucho más potente. Con referencia a los factores de bienestar, Omer (2008) observa que la calidad de vida de un grupo social es normalmente proporcional a su consumo energético. Adicionalmente, varios documentos de las Naciones Unidas también identifican una relación entre el consumo de energía y la calidad de vida (Goldemberg \& Johansson, 1995; Dzioubinski \& Chipman, 1999). En cambio, una revisión bibliográfica en Joyeux \& Ripple (2007) demuestra neutralidad entre el PIB y el consumo energético.

Este trabajo se enfoca en el estudio energético de sistemas urbano-regionales, usando los municipios costeros de la Región Metropolitana de Barcelona como caso de estudio, en el marco de un estudio más amplio sobre sustentabilidad urbana. El sistema elegido demuestra interés por la variedad de los municipios que lo componen, en términos de densidad poblacional y perfil económico. En primer lugar, se realiza el análisis regional del sistema; los indicadores metabólicos derivados se usan para analizar su perfil energético y evaluar su autosuficiencia energética. En una escala más baja, y mediante la desagregación de los datos, el consumo municipal de energía se compara con los índices de densidad de población y de ingreso, con el fin de examinar la validez de los principales patrones y teorías del consumo de energía urbano que se mencionaron anteriormente.

\section{El caso de estudio}

El caso de estudio se compone de 27 municipios que están situados en la costa de Cataluña, una comunidad autónoma española en el noreste de la península ibérica (Fig. 2). La capital de la región es Barcelona, un importante puerto comercial y una de las ciudades más grandes del Mediterráneo. Los municipios seleccionados ocupan $118,6 \mathrm{~km}$ de la costa catalana y tienen un área de $478 \mathrm{~km} 2$, representando el 1,5\% de la superficie de Cataluña. La zona tiene 2,5 millones de habitantes y una densidad mayor a 5000 habitantes por $\mathrm{km} 2$, mientras que el valor promedio equivalente para la Unión Europea en el 2004 era de alrededor de 115 habitantes por km2. La región estudiada se extiende desde el municipio de Malgrat de Mar en el Norte hasta el de Cubelles en el Sur; el municipio de Barcelona está situado en el centro de la zona costera bajo estudio.

La política energética del sistema estudiado es planeada por el Gobierno de Cataluña (Generalitat de Catalunya) y se regula bajo la legislación española y europea. En 2006 el Departamento de Trabajo e Industria del gobierno local (Departament de Treball i Indústria) publicó el Plan Energético de Cataluña (Pla Energètic de Catalunya - PEC) con el fin de regular y establecer objetivos con referencia al manejo de energía en la región. Dicho plan tuvo un enfoque en la gestión sustentable de los recursos energéticos, la optimización del uso energético y la eficiencia y maximización de las fuentes renovables (Generalitat de Catalunya, 2006). La carencia de combustibles fósiles en el sistema lo hace altamente dependiente de las importaciones energéticas; como consecuencia, ciertos tipos de energía primaria, como la nuclear y la eólica, que no están generados localmente pero son comunes en el resto de Cataluña, forman parte del mix energético del sistema. Las principales fuentes primarias de energía que se usan en el sistema estudiado son el petróleo crudo, el gas natural y la energía nuclear, todas importadas y correspondientes al mix energético catalán. 


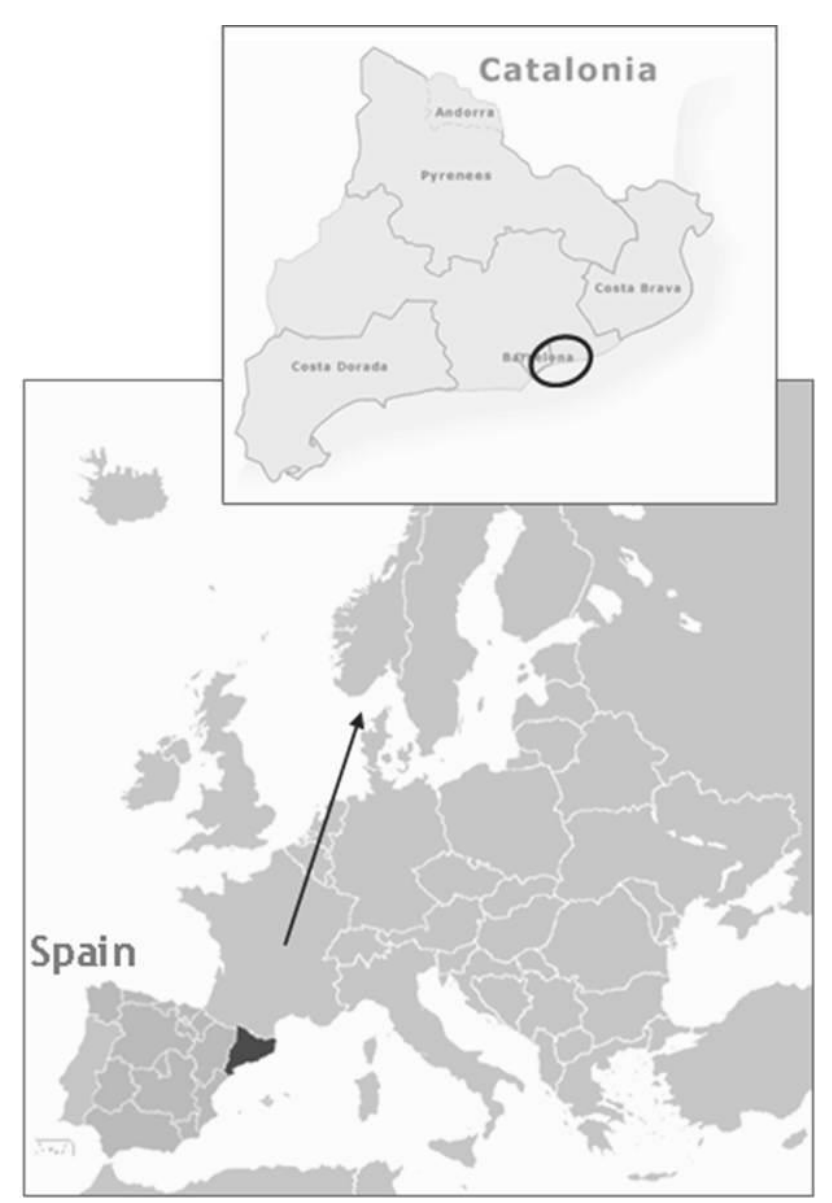

Fig. 2 La ubicación de Cataluña en Europa y los municipios costeros de la Región Metropolitana de Barcelona

Fuente: Elaboración propia

La generación doméstica de electricidad es esencialmente basada en fósiles, los cuales proveen entre el 80 y $100 \%$ de la electricidad generada localmente. Al momento del estudio cuatro plantas térmicas, que funcionaban con fuel oil y gas natural, proveían al sistema de electricidad. De estas, una estaba ubicada en la municipalidad de Cubelles, perteneciente a la Red Eléctrica de España (REE); otra, en la municipalidad de Badalona, bajo la gestión de FECSA-ENHER; y dos reactores más, de la misma compañía, estaban situados en Sant Adriá de Besòs. Estos últimos cerraron en 2003; en 2002 se puso en marcha una planta de ciclo combinado, propiedad de ENDESA S.A.

Otras fuentes de energía generadas locamente, son energía térmica solar y módulos fotovoltaicos en edificios públicos, principalmente en Barcelona; una planta hidroeléctrica de pequeña escala que funciona en el mismo municipio desde el 1999 y plantas de recuperación energética a partir de Residuos Sólidos Urbanos (RSU). Estos últimos incluyen dos plantas de incineración, en Mataró y Sant Adriá de Besòs, la producción de biogás mediante metanización en los Ecoparques de Barcelona y Sant Adriá de Besòs, desde el 2003 y 2006 respectivamente, y la captación de gas del vertedero situado en el municipio de Garraf. Finalmente, una pequeña cantidad de electricidad se genera en unas plantas de tratamiento de aguas residuales (en Besòs, Mataró y El Prat de Llobregat) con cogeneración. Sin embargo, esta energía sirve 
básicamente para la satisfacción de las necesidades de las propias plantas, por lo que no la incluimos en nuestros cálculos.

\section{Marco metodológico}

La metodología usada es adecuada para la simple descripción del metabolismo energético de un sistema regional; esto significa que tanto los flujos indirectos asociados con la generación, distribución y consumo de energía, como la demanda endosomática de energía del sistema se omiten en los cálculos. Adicionalmente, tampoco se considera la eficiencia de cada fuente energética.

Como uno de los objetivos del estudio es evaluar la autosuficiencia energética del sistema, es necesario comparar las necesidades energéticas de este (consumo de energía) con la energía que se genera en su territorio. Como consecuencia, la precisa definición de los límites del sistema, para poder determinar los flujos de entradas y salidas de este, es un paso crucial en la metodología utilizada.

\section{Definición de los límites del sistema}

Con el fin de definir el sistema estudiado se usaron sus fronteras políticas. El territorio elegido en su conjunto no forma una entidad política o administrativamente definida, pero sí los municipios que lo componen. Con referencia a la extracción de los combustibles fósiles y la generación de energía, se usan los límites del sistema como se definen por Eurostat (2001). Según estos, los límites del sistema se definen:

1. Por la extracción de materias primas del medio ambiente local y la emisión/vertido de materiales en este;

2. Por los límites políticos (administrativos) que determinen los flujos materiales desde y hacia otras economías (importaciones y exportaciones).

La energía que se contabiliza es:

- La energía distribuida por la red eléctrica. Esta incluye todas las plantas de generación que proveen al sistema de electricidad, incluyendo las plantas nucleares y las que funcionan con fósiles, más la electricidad importada neta; electricidad generada a partir de fuentes renovables de energía como plantas hidroeléctricas, paneles fotovoltaicos y parques eólicos. La energía solar ocupada para el calentamiento de aguas no se incluye ya que no entra en la red eléctrica y no se puede medir.

- El consumo final de gas natural, propano, butano, todos tipos de diesel (tanto en vehículos como para calefacción, o sea tipos A, B y C) y gasolina, leña y carbón.

- Otros tipos de energía como biomasa o residuos municipales. Los últimos hacen referencia a la electricidad obtenida a partir de la incineración de RSU, el biogás de vertederos y el gas obtenido de procesos de metalización.

\section{Unidades de medida}

Los datos estadísticos usados fueron descritos en varias unidades; sin embargo, el consumo de energía en este trabajo se mide en joules.

\section{Mix de generación eléctrica}

Para calcular el mix eléctrico del sistema consideramos que la electricidad generada localmente se consume por el propio sistema. Principalmente, la generación local se resta de la demanda eléctrica total. Suponemos que el resto, la electricidad importada, es equivalente al mix 
catalán, de acuerdo con otros estudios que se han hecho en similares estudios regionales en la zona (vea por ejemplo Observatori de l'Energia de Barcelona, 2005). La combinación de estos da el mix final del sistema, usando la siguiente ecuación:

$$
x_{T, i}=\left\lfloor\left(x_{R, i} \times E_{R} \div E_{T}\right)+\left(x_{C, i} \times\left(E_{C} \div E_{T}\right)\right\rfloor\right.
$$

Donde,

$\mathbf{X}_{T, \mathbf{i}}$ es la contribución de la fuente primaria $i$ en el mix eléctrico del sistema, en porcentaje

$\mathbf{X}_{\mathbf{R}, \mathbf{i}}$ es la contribución de la fuente primaria $i$ en la generación eléctrica del sistema, en porcentaje

$\mathbf{X}_{\mathbf{C}, i}$ es la contribución de la fuente primaria $i$ en el mix eléctrico catalán, en porcentaje

$\mathbf{E}_{\mathbf{R}}$ es la cantidad de electricidad generado dentro de los límites del sistema, GJ

E c es la cantidad de electricidad importada de la red eléctrica catalana, GJ

$\mathbf{E}_{\mathbf{T}}$ es la cantidad de electricidad total consumida por el sistema, GJ

\section{Producción local de energía}

Todos los portadores de energía usados por el sistema que han sido extraídos dentro de sus límites se consideran como fuentes energéticas locales. En lo que se refiere a las plantas locales de energía que funcionan en base a combustibles fósiles, si estos han sido extraídos localmente la energía generada se considera doméstica. En el caso contrario, o sea cuando las materias primas son importadas, la energía generada se considera importada también.

\section{Importaciones de energía}

Los combustibles fósiles consumidos en y por el sistema, pero que se hayan extraído fuera de sus límites, se consideran importaciones. Lo mismo aplica a las plantas de generación eléctrica; las instalaciones ubicadas en otras regiones que suministran al sistema con electricidad se consideran como importadoras de energía.

\section{Sectores de consumo}

Los datos relacionados con el consumo final de energía se han desagregado con el fin de evaluar e interpretar los resultados de manera más detallada. Esta desagregación también era necesaria para un monitoreo más comprensivo de la economía estudiada. La principal distinción entre sectores es entre los usos domésticos de energía y los demás usos, o sea producción y servicios. Más concretamente, los sectores analizados son los siguientes: (i) sector primario, terciario y transporte, (ii) sector industrial y energético y (iii) sector de construcción y obras públicas.

\section{Fuentes de datos}

Para la realización de este trabajo se necesitaron datos sobre la generación local de energía, el consumo energético final, tanto total como por sector, y finalmente el origen y la contribución de cada materia prima para la generación de electricidad en las plantas locales. Los datos se recolectaron y se organizaron en una base anual y según municipio; la suma de los datos de cada uno de los 27 municipios da una visión agregada al sistema. Esto permite un estudio de la evolución global de las tendencias de consumo, dependencia de importaciones y, al mismo tiempo, la realización de comparaciones entre municipios, en combinación con otros indicadores.

Los datos de generación sobre las plantas de cogeneración se encontraron en los informes anuales que publica la Asociación Española de la Industria Eléctrica (2004; 2007). Con referencia a las energías renovables, solamente se encontraron datos sobre las instalaciones fotovoltaicas de la ciudad de Barcelona en la página web de la Agencia de Energía del municipio (Agència d'Energia de Barcelona, 2008). Los datos de consumo de electricidad y gas natural 
(para el periodo entre 1997 y 2004) fueron facilitados por el Instituto Catalán de Energía después de una comunicación personal. Los datos de consumo final de propano y butano se encontraron por sector, y a nivel de provincia, en la base estadística del Ministerio de Industria, Turismo y Comercio de España (MITYC, 2008). Adicionalmente, los datos necesarios relacionados al consumo final de gasolina y diesel se obtuvieron en la base estadística del Instituto Nacional de Estadística de España (2008).

Para la información que no se hallaba disponible a nivel municipal se usaron métodos de estimación. Las estimaciones más importantes están relacionadas con el consumo de fuel oil, gasolina, butano y propano, durante todo el periodo de estudio, y para el gas natural durante un periodo de tres años (2004-2006). Estos datos se han encontrado a nivel de Comunidad Autónoma (Cataluña) o provincial, y el cálculo de los valores correspondientes del sistema se hizo basándose en las estadísticas de población, disponibles en el Instituto de Estadística de Cataluña (Idescat, 2008a).

\section{Estadísticas e indicadores complementarios}

Con el propósito de interpretar de manera íntegra los resultados relacionados con el consumo energético, se necesitaron ciertas estadísticas e indicadores auxiliares. Algunos de estos eran: (i) Estadística demográfica, (ii) Estadísticas de uso de suelo, (iii) valores de PIB per cápita y (iv) Contribución de cada sector económico al PIB de cada municipio.

\section{Resultados y Discusión}

\subsection{Cálculo del mix eléctrico del sistema}

Dado que el sistema estudiado no forma una entidad política o administrativa, no cuenta con una base estadística, por lo que no se han encontrado datos de consumo primario de energía por fuente. Por otro lado, la búsqueda de datos estadísticos desagregados a nivel municipal ha sido bastante dificultosa, ya que la escala espacial es pequeña y la información encontrada se refería principalmente al consumo energético final. Como consecuencia, el mix eléctrico del sistema se calcula considerando la contribución del mix catalán en las necesidades energéticas del sistema; los resultados se presentan en la Tabla 1.

Las principales tendencias incluyen un aumento estable del gas natural y un descenso del uso de petróleo en la generación de electricidad. También se puede observar la contribución de fuentes energéticas que no se usan dentro del sistema, como la nuclear y los biofósiles. La energía nuclear tiene una contribución decreciente en la generación de electricidad, principalmente debida al lanzamiento de numerosas plantas de ciclo combinado, tanto en el sistema como en la región de Cataluña en su conjunto. Aunque el uso de las energías renovables en Cataluña ha estado aumentando (Ramos-Martín et al., 2007b), su contribución en el mix del sistema está decreciendo, un hecho atribuido al aumento de la generación local de electricidad, la cual se basa en el uso de combustibles fósiles. 
Tabla 1 Evolución anual del consumo energético primario por fuente en el sistema de estudio, en porcentajes $^{\text {a }}$

\begin{tabular}{|l|c|c|c|c|c|c|c|c|c|}
\hline & 1997 & 1998 & 1999 & 2000 & 2001 & 2002 & 2003 & $2004{ }^{\mathrm{b}}$ & $2005 \mathrm{~b}$ \\
\hline Carbón & 1,3 & 0,76 & 0,78 & 0,85 & 0,37 & 0,58 & 0,33 & 0,41 & 0,39 \\
\hline Petróleo & 50,63 & 51,91 & 50,92 & 49,31 & 50,28 & 41,96 & 31,96 & 26,39 & 30,1 \\
\hline Gas natural & 22,95 & 19,42 & 22,4 & 22,44 & 23,97 & 39,77 & 50,44 & 59,21 & 57,57 \\
\hline Nuclear & 21,85 & 23,6 & 20,49 & 21,71 & 19,8 & 13,84 & 12,43 & 9,98 & 7,78 \\
\hline Hidroeléctrica & 1,76 & 1,56 & 1,41 & 1,38 & 1,38 & 0,96 & 1,06 & 0,75 & 0,48 \\
\hline Eólica & 0 & 0 & 0,03 & 0,04 & 0,04 & 0,03 & 0,03 & 0,03 & 0,03 \\
\hline Biomasa & 0,45 & 0,44 & 0,37 & 0,33 & 0,29 & 0,19 & 0,18 & 0,16 & 0,14 \\
\hline Energía solar & 0 & 0 & 0,01 & 0,01 & 0,01 & 0,01 & 0,01 & 0,01 & 0,02 \\
\hline Balance de intercambio de electricidad & 0,43 & 0,7 & 0,77 & 1,1 & 1,17 & 0,62 & 0,65 & 0,34 & 0,89 \\
\hline Residuos renovables & 0,48 & 0,46 & 0,42 & 0,42 & 0,35 & 0,27 & 0,34 & 0,35 & 0,23 \\
\hline Residuos no-renovables & 0,15 & 1,13 & 2,41 & 2,41 & 2,32 & 1,73 & 2,48 & 2,98 & 2,97 \\
\hline Bioetanol & 0 & 0 & 0 & 0 & 0 & 0 & 0,04 & 0,04 & 0,03 \\
\hline Biodiesel & 0 & 0 & 0 & 0 & 0 & 0 & 0,01 & 0,02 & 0,03 \\
\hline Biogás & 0 & 0 & 0 & 0,01 & 0,01 & 0,02 & 0,04 & 0,06 & 0,06 \\
\hline \multicolumn{1}{|c|}{ Total } & 100 & 100 & 100 & 100 & 100 & 100 & 100 & 100 & 100 \\
\hline
\end{tabular}

\subsection{Análisis de la evolución del consumo final de energía por fuente y sector}

La evolución anual del consumo final de energía y la contribución de cada fuente en porcentajes se presentan en el Cuadro 3. La energía producida por fuentes renovables y residuos no-renovables es incluida en la categoría "electricidad", ya que es distribuida por la misma red. Los valores presentados son a partir del año 2000, debido a la falta de datos de consumo de butano, propano, gasolina y diesel para el periodo entre 1997 y 1999 . Es importante destacar el aumento absoluto del consumo de gas natural, incrementando desde casi 33.840 TJ/año en 1997 hasta aproximadamente 81.000 TJ/año en el último año del estudio. Este cambio lo convierte en la principal fuente energética para el sistema en el año 2006, representando el 37,5\% del consumo energético final. De acuerdo al Gas Natural Group (2008), el mayor proveedor de gas natural en España, sus puntos de suministro han aumentado en 1,2 millones de unidades en el estado español durante el transcurso de cuatro años, entre el 2003 y el 2007.

Tabla 2 Consumo energético final, total y per cápita, y contribución por fuente

\begin{tabular}{|l|c|c|c|c|c|c|c|}
\hline & 2000 & 2001 & 2002 & 2003 & 2004 & 2005 & 2006 \\
\hline Electricidad (\%) & 21 & 21,2 & 21,7 & 21,7 & 22 & 19,3 & 19,2 \\
\hline Gas natural (\%) & 24,6 & 25,7 & 24,1 & 25,3 & 24,6 & 36 & 37,5 \\
\hline $\begin{array}{l}\text { Butano } \\
\text { (\%) }\end{array}$ & 1,6 & 1,4 & 1,3 & 1,1 & 1 & 0,8 & 0,7 \\
\hline Propano (\%) & 0,6 & 0,6 & 0,6 & 0,6 & 0,6 & 0,5 & 0,5 \\
\hline $\begin{array}{l}\text { Diesel/fuel oil } \\
\text { (\%) }\end{array}$ & 40,9 & 40,8 & 42 & 42,3 & 43,1 & 36,6 & 36 \\
\hline Gasolina (\%) & 11,3 & 10,2 & 10,2 & 9 & 8,4 & 6,6 & 6,1 \\
\hline $\begin{array}{l}\text { Consumo final total } \\
\text { (TJ/año) }\end{array}$ & 157.055 & 168.266 & 168.366 & 180.676 & 181.433 & 213.953 & 216.652 \\
\hline $\begin{array}{l}\text { Consumo final total } \\
\text { (GJ/cap·año) }\end{array}$ & 67,9 & 72,1 & 70,9 & 73,7 & 73,7 & 85,6 & 85,7 \\
\hline
\end{tabular}


El aumento del consumo de gas natural también explica, y está directamente vinculado, al decrecimiento del consumo de butano, gasolina y diesel, como se puede ver en el Cuadro 3. La transición de estas fuentes energéticas más "tradicionales" al gas natural en los sistemas de calefacción seguramente ha influenciado su consumo. Aunque el valor absoluto de consumo de diesel se ha incrementado durante los años, el tipo C, usado para calefacción, se ha reducido por 50\%, cayendo desde 174.000 a 90.000 toneladas anualmente, entre el 2000 y el 2006 (MITYC, 2008). La contribución del propano y butano es bastante pequeña, sumando menos del $2 \%$ del consumo final de energía durante el tiempo de estudio. Estos gases son usados principalmente en el sector residencial, donde se consume entre el $73 \%$ y el $82 \%$ de su consumo total (MITYC, 2008).

En este punto es relevante recalcar la falta de datos de consumo para carbón y leña a nivel municipal. Sin embargo, la información para toda Cataluña revela una pequeña contribución del carbón en el consumo final de energía para el año 2006, igual al $0.2 \%$ (Instituto Catalán de Energía, 2007). La disminución en el consumo del carbón también se verifica en la caída del número de hogares que usan este combustible para calefacción. Según datos de Idescat (2008a), el porcentaje de hogares en el sistema que usa carbón para este fin ha caído desde un $12,6 \%$ en 1991 a un $0,3 \%$ en 2001. La misma tendencia se observa para la leña, con un descenso de $0,6 \%$ al $0,2 \%$ para el mismo periodo de tiempo.

El consumo de electricidad total y sectorial en valores absolutos se ve mejor ilustrado en la Figura 1, y sus variaciones anuales en la Figura 2; estos datos demuestran un incremento estable de la demanda eléctrica en el sistema durante todo el periodo de estudio, con una tasa anual de crecimiento igual a $4 \%$ y un aumento más moderado durante los últimos tres años de estudio. El sector que más electricidad consume es el de servicios y transporte (Figura 1), lo cual se puede explicar por la economía del sistema estudiado, considerablemente dependiente del sector de servicios. Si tomamos en cuenta que este sector representa básicamente al sector terciario, ya que la agricultura es muy poco desarrollada en el área, este dato revela el gran impacto que tienen los servicios turísticos desarrollados en la zona en su consumo eléctrico. En cambio, el sector de industria y energía demuestra un constante decrecimiento a lo largo de los últimos cuatro años del estudio. Los siguientes datos complementan el comentario anterior; si examinamos la contribución de cada actividad económica al PIB del sistema observaremos una transición de las actividades industriales a las de servicio; en 1991 el sector industrial representaba el $31,2 \%$ de PIB y el sector de servicios el 56,2\% (Idescat, 2008a). Diez años más tarde, el sector industrial bajó por 8 unidades y el de servicios subió por 7, de una manera casi complementaria.

El sector más inestable y menos consumidor en términos energéticos es el de construcción y obras públicas, como se puede ver en ambos gráficos (Fig. 3y Fig. 4). La demanda energética de este sector ha tenido un incremento impresionante entre los años 2002 y 2004 , seguido por una caída en los dos años siguientes. Finalmente, el consumo eléctrico para usos residenciales ha demostrado un crecimiento estable durante todo el periodo de estudio. 


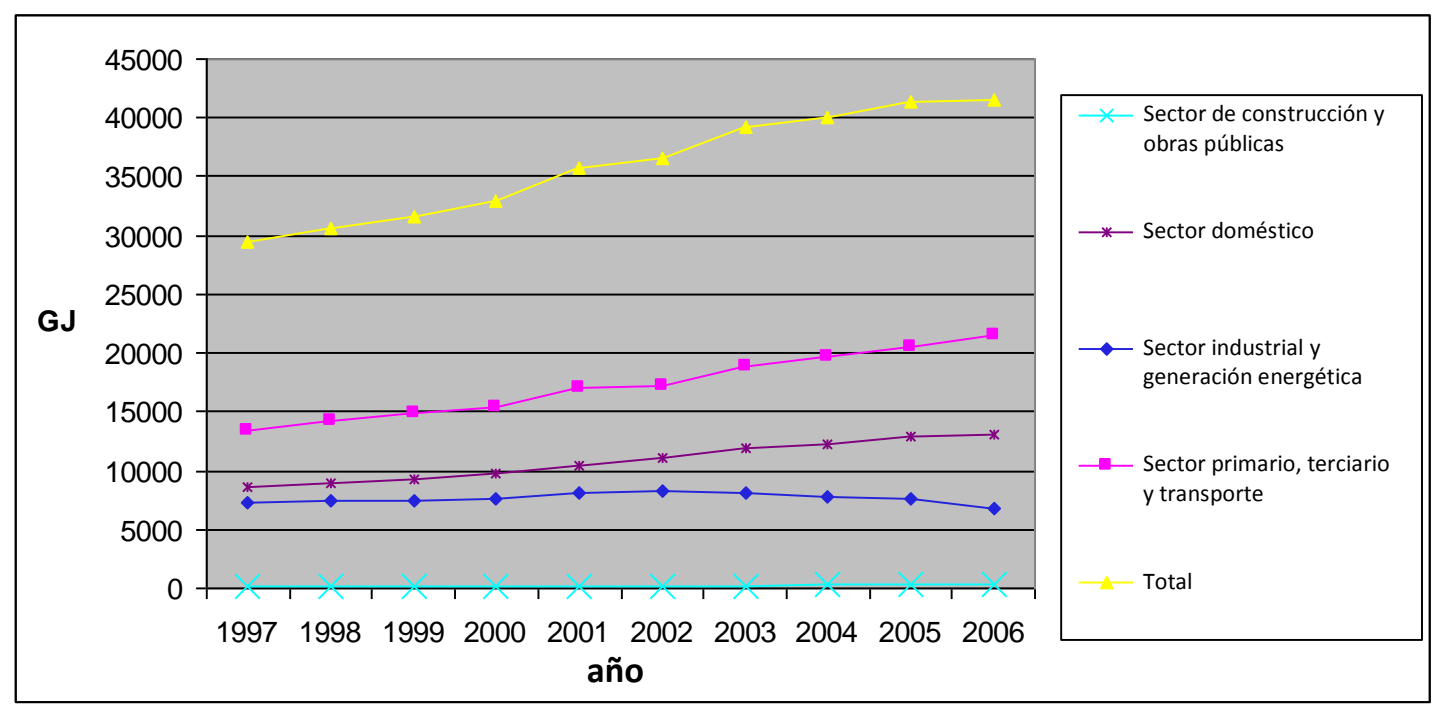

Fig. 3 Consumo final de electricidad por sectores

Fte.: Elaboración propia

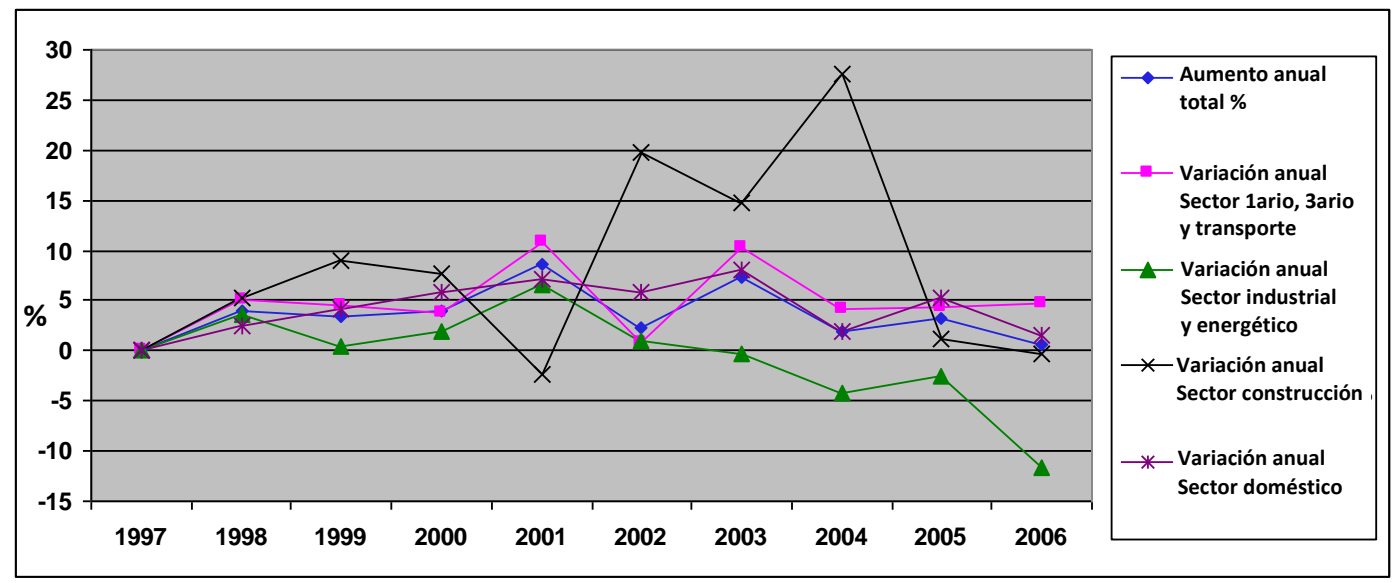

Fig. 4 Variación anual de consumo eléctrico por sectores

Fte.: Elaboración propia

\subsection{Generación local de energía y evaluación de la auto-suficiencia del sistema}

La falta de yacimientos locales de combustibles fósiles limita la generación primaria de energía local a fuentes renovables de energía; los datos oficiales a nivel municipal solo incluyen las instalaciones relevantes en el municipio de Barcelona (Agència d'Energia de Barcelona, 2008). Como se mencionó anteriormente, estos incluyen paneles fotovoltaicos en edificios municipales, una planta mini-hidráulica en el barrio de Trinitat (Barcelona) y las plantas de recuperación energética a partir de RSU. La evolución anual de la generación de energía de estas instalaciones y su contribución al consumo energético total del sistema se resumen en la Tabla 3. 


\section{REVISTA DE \\ URBANISMO}

ISSN 0717-5051

http://revistaurbanismo.uchile.cl
Revista de Urbanismo N³3 - Julio - Diciembre de 2015 Departamento de Urbanismo - FAU - Universidad de Chile

Tabla 3 Generación local de energía primaria, en GJ/año, y su contribución al consumo final de energía y electricidad en el sistema estudiado

\begin{tabular}{|c|c|c|c|c|c|c|c|c|}
\hline & $\begin{array}{c}\text { Paneles } \\
\text { fotovoltaicos } \\
a, b\end{array}$ & $\begin{array}{c}\text { Mini- } \\
\text { hidráulica } \\
b\end{array}$ & $\begin{array}{c}\text { Incineración } \\
R S U \text {, } d\end{array}$ & $\begin{array}{c}\text { Metanización } \\
R S U\end{array}$ & $\begin{array}{c}\text { Vertederos } \\
e, f\end{array}$ & $\begin{array}{c}\text { Generación } \\
\text { total de } \\
\text { energía }\end{array}$ & $\begin{array}{c}\% \text { de } \\
\text { consumo } \\
\text { final de } \\
\text { electricidad }\end{array}$ & $\begin{array}{c}\text { \% de } \\
\text { consumo } \\
\text { final de } \\
\text { energía }\end{array}$ \\
\hline 1997 & - & - & - & - & - & 0 & 0 & 0 \\
\hline 1998 & - & - & 298.199 & - & - & 298.199 & 0,97 & 0,45 \\
\hline 1999 & - & 22.320 & 708.995 & - & - & 731.318 & 2,31 & 1,03 \\
\hline 2000 & 144 & 20.520 & 735.401 & - & - & 756.065 & 2,3 & 0,48 \\
\hline 2001 & 288 & 18.720 & 773.752 & - & - & 792.760 & 2,22 & 0,47 \\
\hline 2002 & 738 & 22.680 & 592.240 & 9.572 & - & 625.234 & 1,71 & 0,37 \\
\hline 2003 & 932 & 21.960 & 931.968 & 22.349 & - & 977.213 & 2,49 & 0,54 \\
\hline 2004 & 2.930 & - & 862.517 & 62.413 & 292.194 & 1220.058 & 3,05 & 0,67 \\
\hline 2005 & 2.930 & - & 898.654 & 23.576 & 292.194 & 1217.354 & 2,95 & 0,57 \\
\hline 2006 & 2.930 & - & 317.225 & 588.269 & 187.441 & 1095.865 & 2,64 & 0,51 \\
\hline
\end{tabular}

a Datos calculados a partir de la potencia instalada

b Fuente: Canal Solar BCN (2008)

c La energía generada en la planta de Mataró se calculó a partir de la cantidad de los residuos tratados y el coeficiente de generación de energía, igual a $1.894 \mathrm{GJ} / \mathrm{t}$ (Fuente: Consortium for the Management of Solid Municipal Waste in Maresme, 2008)

' Los datos de generación de energía en la planta incineradora de Sant Adriá de Besòs fueron facilitados por Tratamiento y Selección de Residuos, S.A. (2008)

e Los datos sobre las plantas de metanización y los vertederos se encontraron en los informes anuales emitidos por la Autoridad Metropolitana Ambiental de Barcelona (Entitat Metropolitana del Medi Ambient, $2004 ; 2005 ; 2006 ; 2007$ )

f Se refiere a la energía obtenida del gas de vertedero

Aparentemente, la contribución de estas fuentes a las necesidades energéticas del sistema es trivial, llegando a una contribución máxima anual de $3 \%$ y $1 \%$ al consumo final de electricidad y energía total respectivamente. Al contrario de la situación general en Cataluña en su conjunto, y con España siendo el segundo generador más importante de energía eólica en la Unión Europea, el sistema no cuenta con ningún parque eólico. Aunque es un área compatible con la instalación de parques eólicos (Generalitat de Catalunya, 2008), su potencial no es muy prometedor (Asociación Eólica de Catalunya, 2008). Del mismo modo, el desarrollado sector de servicios que domina la economía del sistema y su extensa urbanización pueden explicar la falta de producción de biocombustibles; la producción agrícola en la zona es muy baja, y como consecuencia no hay cultivos dedicados a fines energéticos.

A pesar de que todas las fuentes primarias de energía son importadas, la generación de electricidad en las plantas locales es bastante elevada. La Fig. 5 Evolución anual de la generación doméstica de electricidad y el consumo total de electricidad muestra la evolución anual de la generación local de electricidad3 comparada con la demanda final. Su contribución oscila desde un promedio de 21,4\% para el periodo entre 1997 y 2001, hasta el 53,4\% para los años siguientes. El valor más alto se observa el año 2005, cuando las plantas locales cubrieron el $60 \%$ de las demandas del sistema en electricidad.

\footnotetext{
${ }^{3}$ Con referencia a todas las plantas locales, independientemente del origen de las materias primas
} 


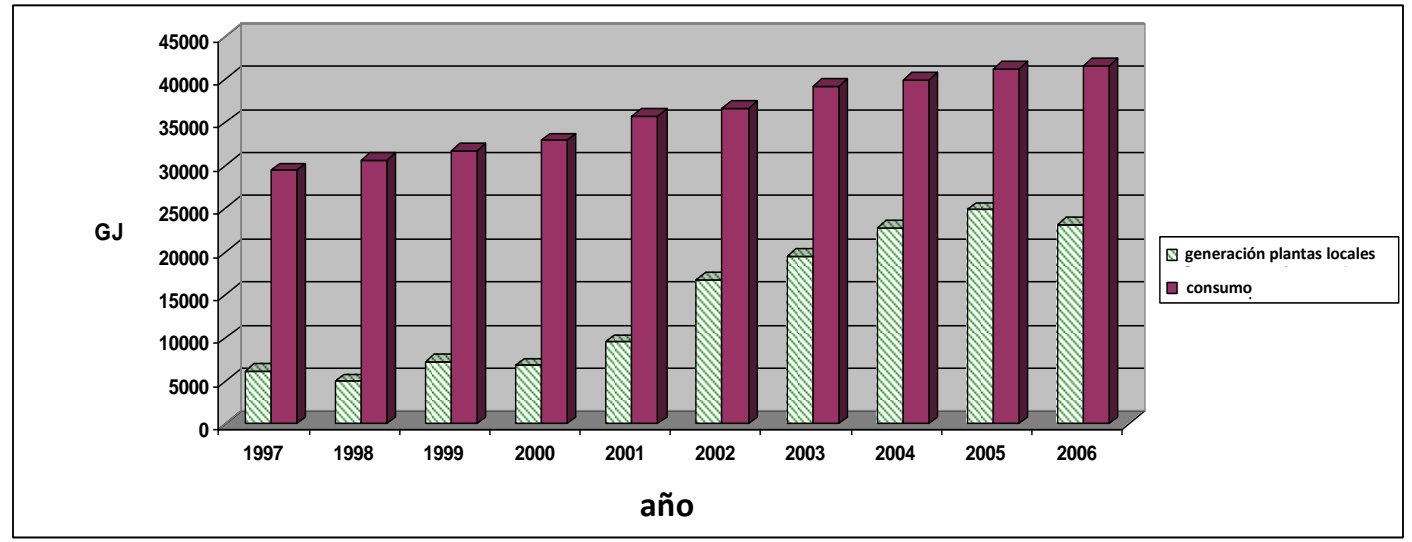

Fig. 5 Evolución anual de la generación doméstica de electricidad y el consumo total de electricidad Fte.: Elaboración propia

El repentino incremento de generación eléctrica que se observa para el año 2002 se atribuye al lanzamiento de dos unidades de una planta de ciclo combinado en el municipio de Sant Adriá de Besòs, lo cual puede observarse más claramente en la Fig. 6 Evolución anual de la generación local de electricidad por fuente. Los datos del mismo gráfico demuestran que la generación local de electricidad se caracteriza por el predominio de las instalaciones de gas natural, una pequeña contribución de procesos de recuperación energética de RSU y la contribución de fuentes renovables.

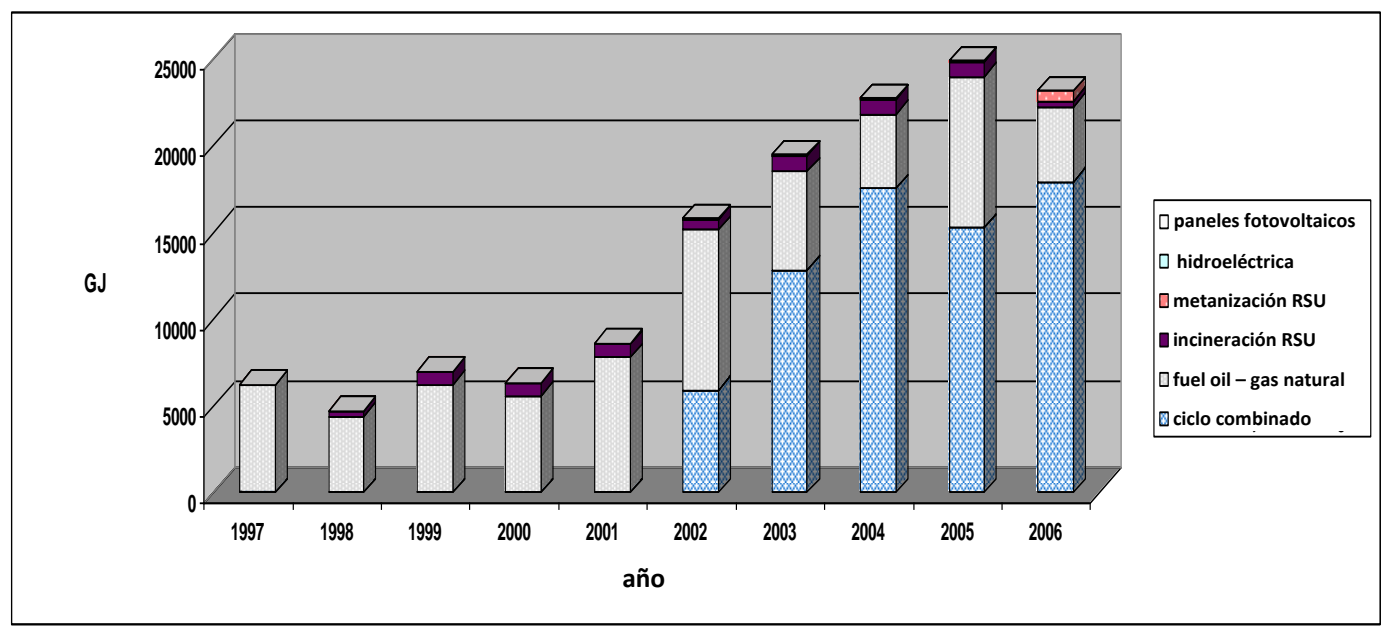

Fig. 6 Evolución anual de la generación local de electricidad por fuente Fte.: Elaboración propia

\subsection{Análisis del consumo energético a nivel municipal}

En esta sección se presentan los resultados de la comparación de los derivados indicadores de consumo energético con datos de PIB y densidad de población, a nivel municipal. El objetivo es revelar posibles patrones de consumo asociados a distintos tipos de economías y tipos de urbanización. Para dicho análisis se usaron datos de los municipios con población superior a 5.000 habitantes y para el año 2001. Debido a la falta de datos desagregados de consumo energético, este análisis comparativo se hizo en base al consumo final de electricidad por sector y total, y el consumo total de gas natural. 


\section{REVISTA DE \\ URBANISMO}

ISSN 0717-5051

http://revistaurbanismo.uchile.cl
Revista de Urbanismo N³3 - Julio - Diciembre de 2015 Departamento de Urbanismo - FAU - Universidad de Chile

Como se mencionó anteriormente, tanto el consumo de electricidad como el PIB se consideran indicadores de calidad de vida, de modo que se tendría que esperar una correlación positiva entre los dos. No obstante, los municipios del caso de estudio no demuestran una alta diversidad de valores de PIB, lo cual proporcionaría un análisis interesante y conclusiones valiosas en este aspecto. Sin embargo, los datos en la Fig. 7 sí revelan una conexión positiva en los casos de consumo total de electricidad y el sector de transporte y servicios.

La municipalidad del Prat de Llobregat, una zona altamente industrializada situada al lado de Barcelona, es el municipio que presenta el más alto valor de PIB per cápita. Sus elevadas demandas energéticas se deben a las actividades industriales, considerando el relativamente bajo consumo en los sectores residencial y de construcción. Si damos por sentado que el consumo de electricidad doméstico es correlacionado al PIB, entonces el Prat de Llobregat se podría caracterizar como un rico municipio industrial con habitantes de bajos ingresos.

Otro caso interesante es el de Sitges, un centro turístico que presenta valores de consumo sorprendentemente similares a los del Prat de Llobregat en Fig. 7b, Fig. 7d y Fig. 7f. Esto se puede atribuir a dos factores; el elevado consumo en los sectores residencial, de servicios y construcción se puede explicar por la economía del municipio, basada en el turismo, que tiene como consecuencia altas inversiones en dichos sectores, y el aumento temporal de su población por un $24 \%$ (Idescat, 2008b). Con referencia al consumo industrial, esto se puede atribuir a la baja densidad de habitantes de Sitges, que aumenta los valores per cápita. Finalmente, la ciudad de Barcelona tiene relativamente moderados valores per cápita, con la excepción de los sectores de servicios y transporte, debido a la gran cantidad de visitantes que la ciudad recibe y el funcionamiento de una red de tranvía eléctrico.

Por lo que se refiere a la densidad de población, no se manifiesta una tendencia general de correlación con los indicadores de consumo energético que se estudian aquí (Fig. 8). No obstante, destaca una clara correlación negativa con el consumo eléctrico en los sectores residencial y de construcción (Fig. 8c y Fig. 8e). Con referencia al último sector, si consideramos que estos municipios están siendo urbanizados al mismo ritmo, es esperable que los sistemas que son densamente poblados demuestren valores de consumo per cápita más bajos.

Finalmente, y con respecto a los resultados sobre la relación entre la superficie urbanizada de un municipio y sus necesidades energéticas, estos son análogos a los presentados para la densidad de población. Estos dos factores tienen una correlación positiva, ya que la alta densidad poblacional está vinculada a sistemas extensamente urbanizados. 


\section{REVISTA DE \\ URBANISMO}

ISSN 0717-5051

http://revistaurbanismo.uchile.cl
Revista de Urbanismo N³3 - Julio - Diciembre de 2015

Departamento de Urbanismo - FAU - Universidad de Chile

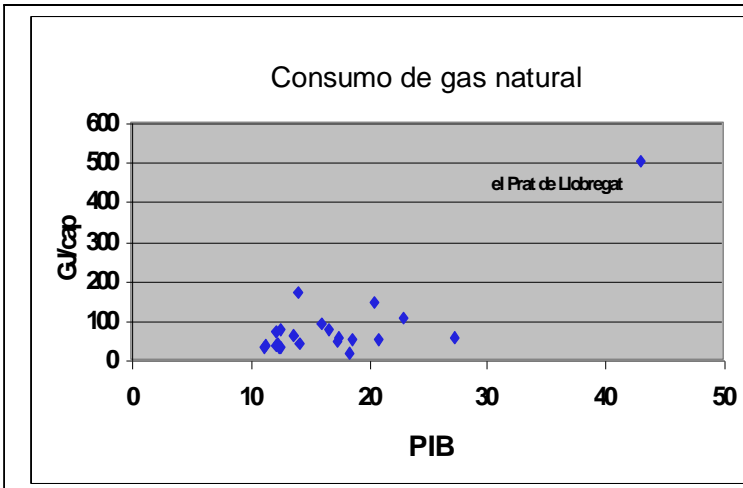

(a)

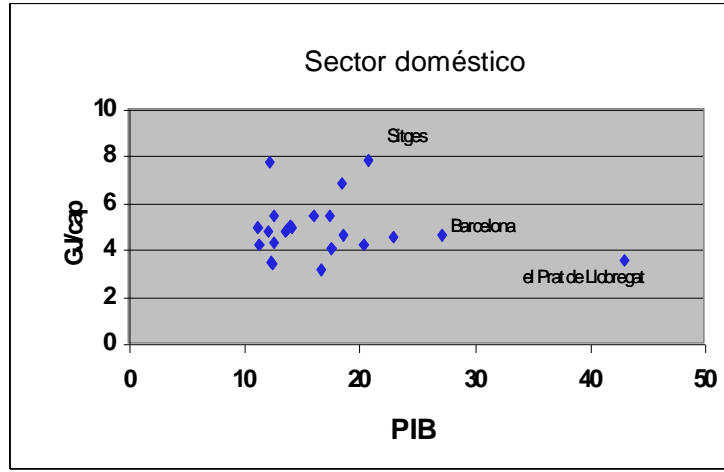

(c)

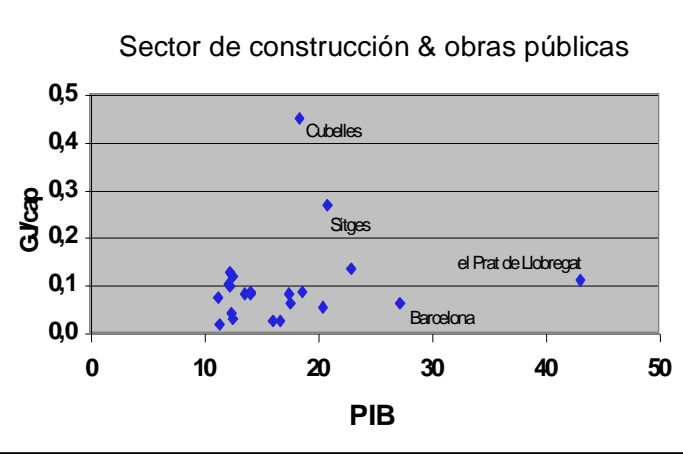

(e)

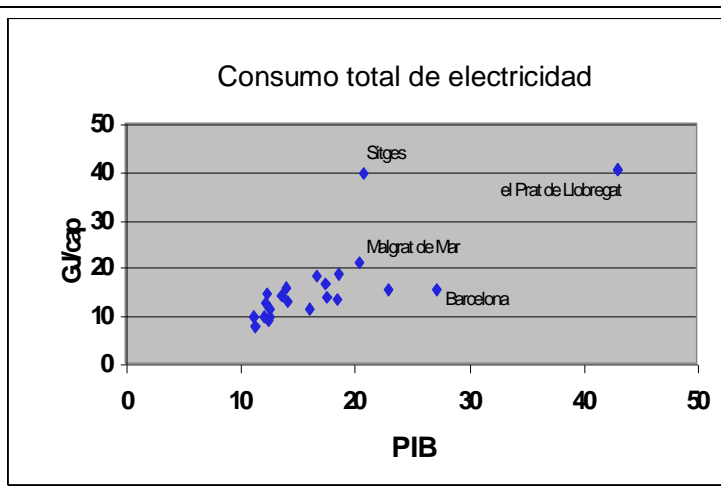

(b)

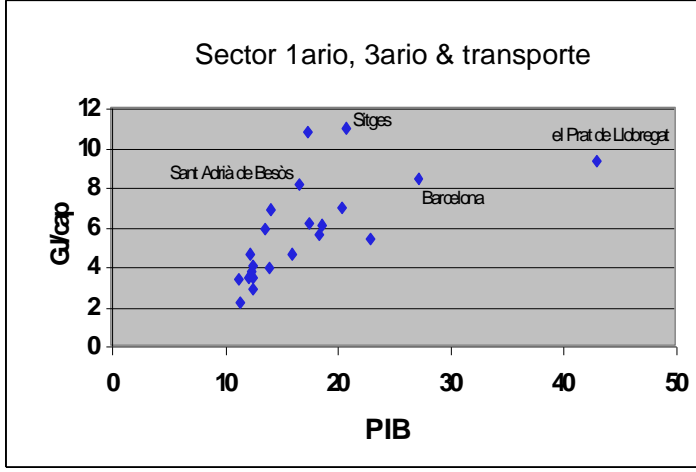

(d)

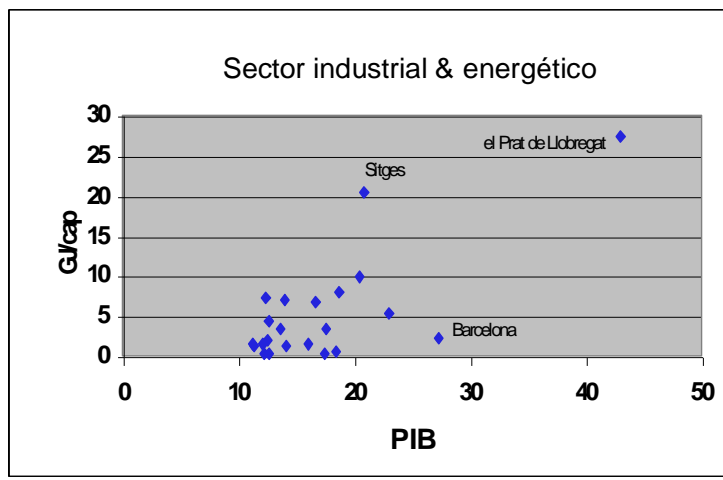

(f)

Fig. 7 Correlación entre el PIB (en miles de euros per cápita) y principales indicadores de consumo energético, para el 2001

(a) consumo anual de gas natural (b) consumo total de electricidad (c) consumo de electricidad para uso doméstico (d) consumo de electricidad para servicios y transporte (e) consumo de electricidad para la construcción y obras públicas (f) consumo de electricidad para usos industriales y la generación de energía 


\section{REVISTA DE \\ URBANISMO}

ISSN 0717-5051

http://revistaurbanismo.uchile.cl
Revista de Urbanismo N³3 - Julio - Diciembre de 2015

Departamento de Urbanismo - FAU - Universidad de Chile

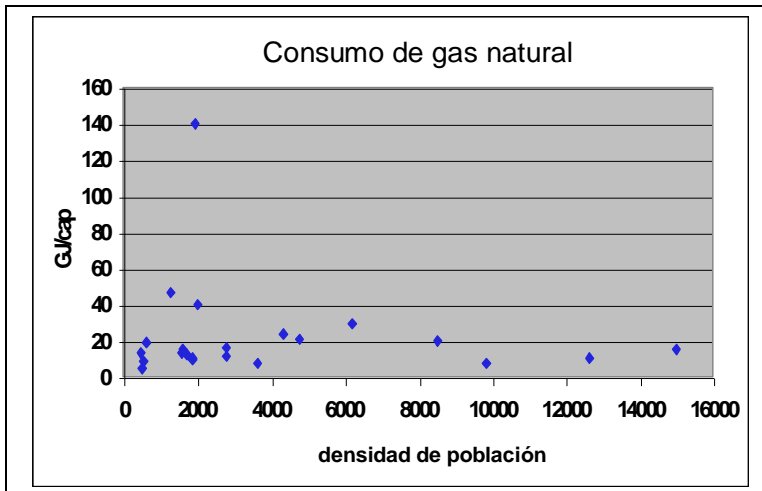

(a)

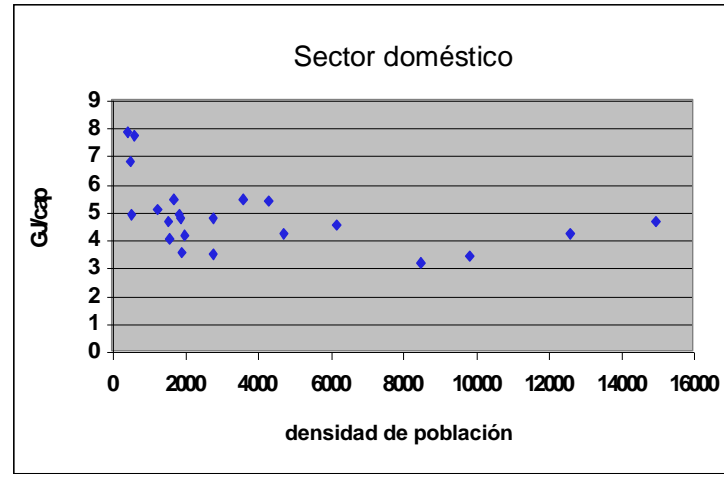

(c)

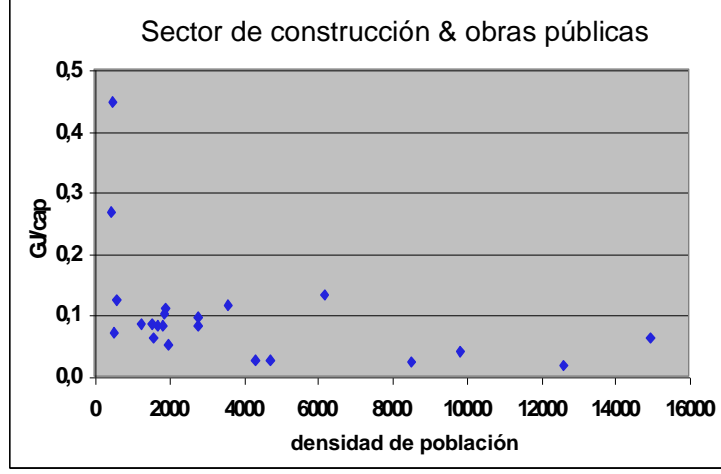

(e)

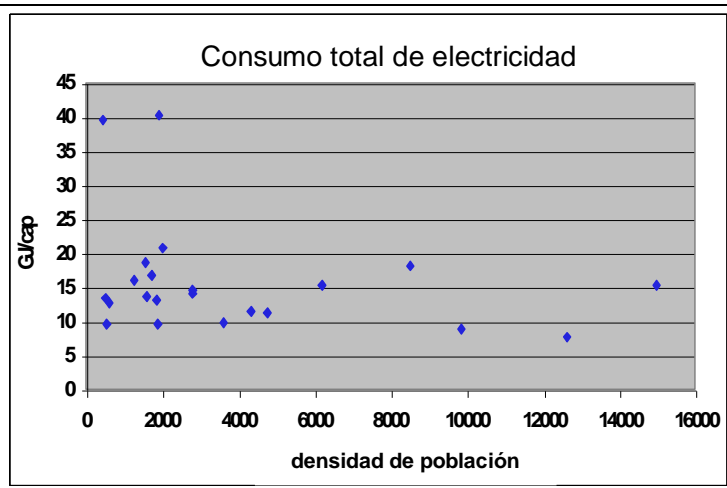

(b)

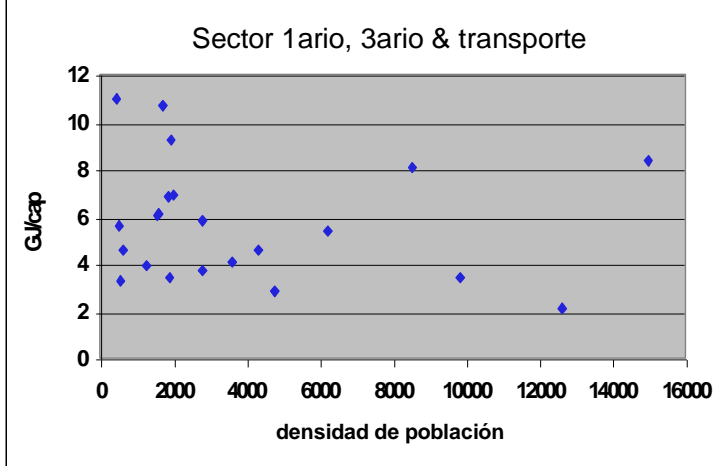

(d)

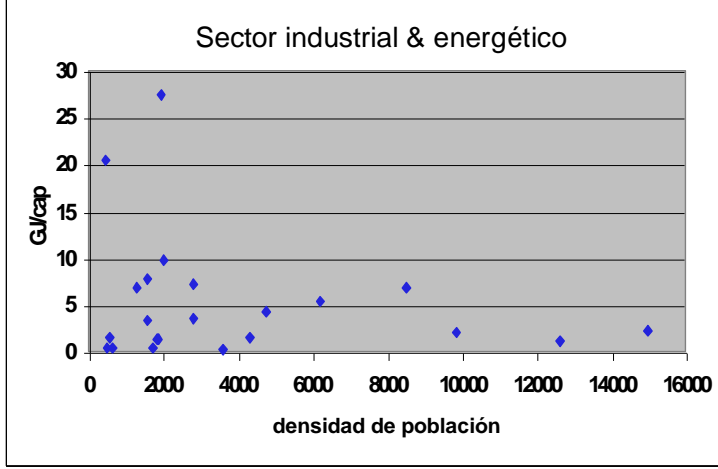

(f)

Fig. 8 Correlación entre la densidad de población, en habitantes por km2, y principales indicadores de consumo energético, en GJ per cápita, para el 2001

(a) consumo anual de gas natural (b) consumo total de electricidad (c) consumo de electricidad para uso doméstico (d) consumo de electricidad para servicios y transporte (e) consumo de electricidad para la construcción y obras públicas ( $f$ ) consumo de electricidad para usos industriales y la generación de energía 


\section{Conclusiones}

Este artículo examinó la utilidad del análisis energético como una herramienta para los estudios urbanos y regionales. Con este fin, se propuso un simple esquema metodológico para la cuantificación del metabolismo energético. La metodología se aplicó a dos escalas geográficas (regional y municipal) de un sistema urbanizado, en una zona costera del Mediterráneo. Los indicadores obtenidos tuvieron como objetivo indagar en la evaluación de la autosuficiencia energética del sistema y comprobar la sensibilidad de la correlación entre el consumo energético y la productividad según sectores económicos, la densidad urbana y la calidad de vida de sus habitantes.

En primer lugar, el análisis temporal indicó que el consumo energético final del sistema costero bajo estudio se ha ido incrementando durante los últimos años, tanto en valores absolutos como per cápita. Debido a la falta de yacimientos locales de combustibles fósiles y la baja generación de energías renovables, se demuestra que la región es altamente dependiente de las importaciones energéticas. Lo mismo aplica para el sector eléctrico; a pesar del aumento de la generación de electricidad en las plantas locales, estas siguen funcionando con materias primas importadas, limitando la autosuficiencia energética del sistema.

Esta última observación hace evidente la relevancia del análisis energético con una perspectiva sistémica, como la del metabolismo energético a nivel regional. Dicho análisis revela la real autosuficiencia del sistema, viendo más allá de la generación de energía local y considerando la procedencia de las materias primas. En este sentido, si bien en el caso de estudio se llegó a generar más del $50 \%$ de la electricidad requerida, la cantidad de esta que fue producida por fuentes primarias locales, apenas logró superar el $3 \%$ de la demanda total. El bajo aprovechamiento de las energías renovables contribuye substancialmente en esta dependencia.

Otro resultado relevante es que el consumo final de fuentes energéticas más tradicionales como la gasolina, el petróleo, el butano y el diesel han dado lugar al gas natural. Sin embargo, esta transición no ha mejorado ni el grado de dependencia del sistema a las importaciones ni su sustentabilidad, ya que se mantiene el predominio del uso de un combustible fósil importado. La conducción de estudios más específicos en torno a este tema sería interesante para la revelación de los aspectos medioambientales de esta transición, como un posible cambio en la emisión total de CO2 usando por ejemplo la metodología del Análisis de Ciclo de Vida.

Con referencia a la investigación de nexos entre consumo energético urbano y densidad poblacional, los resultados verifican la tesis que vincula las ciudades de baja densidad con un incremento del consumo energético en el sector doméstico y el de construcción. Asimismo, los resultados demuestran que altos valores de PIB per cápita, como índice de calidad de vida, están relacionados positivamente al consumo total de electricidad y a la demanda eléctrica en los sectores de servicios y transporte. Como consecuencia, y debido al costo que tiene la dispersión urbana, en términos de consumo energético y sostenibilidad ambiental, debe gestionarse una planificación urbana que tienda a la ciudad compacta. De esta forma, se reduciría la necesidad de transporte particular, el consumo energético y las emisiones de $\mathrm{CO}_{2}$, puesto que se ha demostrado que las políticas de control de la expansión urbana pueden limitar el uso del automóvil (Handy et al., 2005).

El análisis energético sectorial reveló que el perfil económico de cada municipio es un factor importante en la determinación de patrones de consumo energético. Los municipios con actividad turística demuestran un elevado consumo de electricidad per cápita en los sectores doméstico y de servicios, como consecuencia del aumento temporal de la población y de la infraestructura asociada, correspondientemente. 
De lo anterior se puede deducir que el estudio del metabolismo energético a escala urbana y regional es una herramienta que no solo revela datos interesantes para la evolución de los territorios estudiados y la interrelación de sus economías con el uso de la energía; la investigación más extensa y profunda a nivel urbano puede resultar en concretas propuestas de planificación con el fin de ahorrar recursos y alcanzar el objetivo de la autosuficiencia energética. Esto verifica que la planificación territorial y el urbanismo tienen un rol fundamental en la generación de una ciudad más eficiente energéticamente, y con ello sustentable.

La importancia de estas conclusiones no es menor para la realidad chilena. Como a nivel nacional la contribución de las energías renovables en la generación de electricidad es trivial y el sector del transporte está alimentado exclusivamente por combustibles fósiles, la forma de la urbe chilena también contribuye inevitablemente en la emisión de CO2, uno de los principales denominados Gases de Efecto Invernadero, causa del cambio climático. En este contexto, las ciudades son tanto promotores del cambio climático, como lugares donde se espera ver los impactos socio-ambientales debidos a los cambios de temperatura y la disminución de recursos naturales como el agua (Romero Lankao y Qin, 2011). La manera de afrontar a estos cambios es mediante estrategias de mitigación y adaptación (Barton, 2009). Repensar la forma urbana con el fin de aumentar la eficiencia energética de un asentamiento humano es una de las formas de mitigación al cambio climático, ya que la reducción del consumo energético restringirá la emisión de $\mathrm{CO} 2$.

Finalmente, y con respecto a la política energética nacional de los últimos años, los efectos de ella han demostrado que las grandes infraestructuras de generación eléctrica causan malestar social y son fuentes de conflictos socio-ambientales. El acercamiento al tema energético y, más concretamente, el de la soberanía energética nacional, tiene que empezar por diagnósticos a las escalas geográficas más bajas. El aprovechamiento de los recursos locales y renovables y la óptima planificación territorial son elementos necesarios para una política energética nacional multi-escalar que contribuya al desarrollo local en un contexto de cambios globales.

\section{Referencias}

Agència d'Energia de Barcelona. (2005). El comptador. Informació Energética de Barcelona. (Num.1) Barcelona: Autor.

Agència d'Energia de Barcelona. (2008). Centrales fotovoltaicas. Extraído el 8 Enero, 2010 de http://www.barcelonaenergia.cat/cas/observatorio/fotovolt/resumefv.htm

Agencia Europea de Medio Ambiente. (1999). Environment in the European Union at the turn of the century. Environmental assessment report no. 2. Office for Official Publications of the European Union. European Environment Agency: Luxembourg.

Agencia Internacional de Energía. (1995). Energy balances of OECD countries 1992-1993. París: Autor.

Agencia Internacional de Energía. (2008). Statistics \& Balances. Extraído el 8 Diciembre, 2008 de http://www.iea.org/stats/index.asp

Asociación Eólica de Catalunya. (2008). Areas with wind potential in Catalonia. EolicCat, l'Associació Eòlica de Catalunya. Extraído el 16 Mayo, 2010 de http://www.eoliccat.net/_docs/zones.asp

Asociación Española de la Industria Eléctrica. (2004). Memoria Estadística Eléctrica de UNESA. 2004. Asociación Española de la Industria Eléctrica. Extraído el 18 Enero 2009, de www.unesa.es

Asociación Española de la Industria Eléctrica. (2007). Memoria Estadística Eléctrica de UNESA. 2007. Asociación Española de la Industria Eléctrica. Extraído el 18 Enero 2009, de www.unesa.es 
Barton, J. R. (2009) Adaptación al cambio climático en la planificación de ciudades-regiones. EURE, 43, 530.

Brugmann, J. (1996). Planning for sustainability at the local government level. Environmental Impact Assessment Review, 16, 363-379.

Canal Solar BCN (2008). http://www.barcelonaenergia.cat/canalsolar/

Consortium for the Management of Solid Municipal Waste in Maresme (2008). Consorci per al Tractament de Residus Sòlids Urbans del Maresme. http://www.plantabrossa-maresme.com/infocaps.php?id=1234

Dzioubinski, O. \& Chipman, R. (1999). Trends in consumption and production: household energy consumption. DESA Discussion Paper No. 6, Economic and Social Affairs, United Nations.

Entidad Metropolitana del Medio Ambiente. (2004). Metropolitan Environmental Data 2003. Barcelona: Entitat Metropolitana dels Serveis Hidràulics i del Tractatament de Residus (EMSHTR).

Entidad Metropolitana del Medio Ambiente. (2005). Metropolitan Environmental Data 2004. Barcelona: Entitat Metropolitana dels Serveis Hidràulics i del Tractatament de Residus (EMSHTR).

Entidad Metropolitana del Medio Ambiente. (2006). Metropolitan Environmental Data 2005. Barcelona: Entitat Metropolitana dels Serveis Hidràulics i del Tractatament de Residus (EMSHTR).

Entidad Metropolitana del Medio Ambiente. (2007). Metropolitan Environmental Data 2006. Barcelona: Entitat Metropolitana dels Serveis Hidràulics i del Tractatament de Residus (EMSHTR).

España, Instituto Nacional de Estadística. (2008). www.ine.es

España, Ministerio de Industria, Turismo y Comercio de España [MITYC] (2008). Estadísticas y Balances Energéticos - Publicaciones Estadísticas Gas. Ministerio de Industria, Turismo y Comercio. Extraído el 28 Mayo, 2009 de http://www.mityc.es/Balances/Seccion/Publicaciones/Estadisticas/Gas/

Eurostat (1999). Towards environmental pressure indicators for the EU. Luxembourg: Office for Official Publications of the European Communities.

Eurostat (2001). Economy-wide material flow accounts and derived indicators. A methodological guide. Luxembourg: Office for Official Publications of the European Union.

Fischer-Kowalski, M. (1998). Society's metabolism. The intellectual history of materials flow analysis. Part I: 1860-1970. Journal of Industrial Ecology, 2 (1), 61-78.

Fischer-Kowalski, M. \& Haberl, H. (1998). Sustainable development: socio-economic metabolism and colonization of nature. International Social Science Journal, 50 (4), 573-587.

Gas Natural Group (2008). Operating and Financial Statistics. Disponible en: http://portal.gasnatural.com/archivos/informesPDF/280508_Estadisticas_de_explotacion_financieras_ing. pdf

Gasol, C. M., Martínez, S., Rigola, M., Rieradevall, J., Anton, A., Carrasco, J., Ciria, P. \& Gabarrell, X (2009). Feasibility assessment of poplar bioenergy systems in the Southern Europe. Renewable and Sustainable Energy Reviews, 13 (4), 801-812.

Generalitat de Catalunya (2006). Pla de I' Energia de Catalunya 2006-2015. Generalitat de Catalunya. Barcelona: Departament de Treball i Indústria.

Generalitat de Catalunya (2008). Ministry of the Environment: Pla de Parcs eòlics de Catalunya. Disponible en: http://mediambient.gencat.net

Giacobbe, N., Frediani, J., Aon, L. \& Ravella, O. (2007) El consumo energético del sector transporte analizado desde la demanda. Aspectos metodológicos. Facultad de Arquitectura y Urbanismo, Instituto de 
Estudios del Hábitat, Unidad de Investigación. ASADES, Avances en Energías Renovables y Medio Ambiente, Vol. 11, Argentina. ISSN 0329-5184

Goldemberg, J. \& Johansson, T.B. (eds.) (1995). Energy as an instrument of socio-economic development. United Nations Development Programme. Extraído el 18 Abril 2009 de http://www.undp.org/seed/energy/policy

Haberl, H. (2001). The energetic metabolism of societies. Part I: Accounting concepts. Journal of Industrial Ecology, 5 (1), 11-33.

Haberl, H. (2006). The global socioeconomic energy metabolism as a sustainability problem. Energy, 31, 87-99.

Haberl, H., Fischer-Kowalski, M., Krausmann, F., Weisz, H. \& Winiwarter, V. (2004). Progress towards sustainability? What the conceptual framework of material and energy flow accounting (MEFA) can offer. Land Use Policy, 21, 199-213.

Handy, S., Cao, X. \& Mokhtarian, P. (2005) Correlation or Causality between the Built Environment and Travel Behavior? Evidence from Northern California. Transportation Research D, 10: 427-444

Huang, S.-L. (1998). Urban ecosystems, energetic hierarchies, and ecological economics of Taipei metropolis. Journal of Environmental Management, 52, 39-51.

Huang, S.-L., Kao, W.-C. \& Lee, C.-L. (2007). Energetic mechanisms and development of an urban landscape system. Ecological Modelling, 201 (3-4), 495-506.

Instituto Catalán de Energía. (2007). Balanç energètic de Catalunya 2006. Institut Català d' Energia. Generalitat de Catalunya. Setmana de l'energia 2007. Extraído el 19 Octubre, 2007 de http://www.gencat.cat/economia/doc/doc_29565278_1.pdf

Instituto de Estadística de Cataluña [Idescat]. (2008a). Institut d'Estadística de Catalunya. Bases de datos disponibles en: www.idescat.cat

Instituto de Estadística de Cataluña [Idescat]. (2008b). Database of municipalities and counties: Sitges. Institut d'Estadística de Catalunya. Extraído el 19 Febrero, 2008 de http://www. idescat.cat/territ/BasicTerr?TC $=5 \& V 0=1 \& V 3=863 \& V 4=435 \&$

ALLINFO $=$ TRUE\&PARENT $=1 \& C T X=B \& V 1=08270 \& V O K=$ Confirmar

Joyeux, R \& Ripple, R. D. (2007). Household energy consumption versus income and relative standard of living: A panel approach. Energy Policy, 35, 50-60.

Kastoriadis, C. \& Cohn-Bendit, D. (1981). From ecology to autonomy. Atenas: Kedros publishing.

Kennedy, C, Cuddihy, J. \& Engel-Yan, J. (2007). The changing metabolism of cities. Journal of Industrial Ecology, 11 (2), 1-17.

Krausmann, F. \& Haberl, H. (2002). The process of industrialization from the perspective of energetic metabolism. Socioeconomic energy flows in Austria 1830-1995. Ecological Economics, 41, 177-201.

Krausmann, F., Haberl, H., Erb, K.-H. \& Wackernagel, M. (2004). Resource flows and land use in Austria 1950-2000: using the MEFA framework to monitor society-nature interaction for sustainability. Land Use Policy 21, 215-230.

Larivière, I. \& Lafrance, G. (1999). Modelling the electricity consumption of cities: effect of urban density. Energy Economics, 21 (1), 53-66.

Martinez-Alier, J. (1987). Ecological economics: energy, environment and society. Oxford: Basil Blackwell. Martinez-Alier, J. (2004). Marx, Energy and social metabolism. Encyclopaedia of Energy, 3, 825-834. 


\section{REVISTA DE \\ URBANISMO}

ISSN 0717-5051

http://revistaurbanismo.uchile.cl
Revista de Urbanismo N³3 - Julio - Diciembre de 2015

Departamento de Urbanismo - FAU - Universidad de Chile

Matthews, E., Amann, C., Bringezu, S., Fischer - Kowalski, M., Hüttler, W., Kleijn, R. et al. (2000). The Weight of Nations: Material Outflows from Industrial Economies. Washington: World Resources Institute.

Mindali, O., Raveh, A. \& Salomon, I. (2004). Urban density and energy consumption: a new look at old statistics. Transportation Research Part A, 38, 143-162.

Naciones Unidas. (1997). Energy statistics yearbook 1995. Nueva York: Autor.

Naciones Unidas - Departamento de Asuntos Económicos y Sociales. (2001). Indicators of Sustainable Development: Guidelines and Methodologies, 2nd edition, September. New York: Autor.

Newman, P. \& Kenworthy, J. (1989). Cities and Automobile Dependence: An International Sourcebook. Londres: Avebury Technical.

Observatori de l'energia de Barcelona (2005). Observatori de L'energia de Barcelona. Balanç 1999 2003. Barcelona: Autor.

Oliver-Solà, J., Núñez, M., Gabarrell, X., Boada, M. \& Rieradevall, J. (2007). Services sector metabolism, Integrated Energy Flow Analysis and Life Cycle Analysis of the technical energy consumption at the Montjuic Park. Journal of Industrial Ecology, 11(2), 83-98.

Omer, A., M. (2008). Energy, environment and sustainable development. Renewable and Sustainable Energy Reviews, 12 (9), 2265-2300.

Organismo Internacional de Energía Atómica. (2005). Energy Indicators for Sustainable Development: Guidelines and Methodologies. Vienna: Autor.

Ortiz, J. \& Morales, S. (2002) Impacto socioespacial de las migraciones intraurbanas en entidades de centro y de nuevas periferias del Gran Santiago. EURE, 28(85), 171-185.

Ramos-Martín, J., Giampietro, M. \& Mayumi, K. (2007a). On China's exosomatic energy metabolism: An application of multi-scale integrated analysis of societal metabolism (MSIASM). Ecological Economics, 63, 174-191.

Ramos-Martín, J., Cañellas Boltà, S. \& Russi, D. (2007b). Anàlisi del Metabolisme Energètic de l'Economia Catalana (AMEEC). Bloc 2: Energia Primària a Catalunya. Resum executiu. Consell Assessor pel Desenvolupament Sostenible (CADS). Barcelona: Generalitat de Catalunya.

Romero, H. \& Vásquez, A. (2005) La comodificación de los territorios urbanizables y la degradación ambiental en Santiago de Chile. Scripta Nova, 9 (194)

Romero Lankao, P. \& Qin, H. (2011) Conceptualizing urban vulnerability to global climate and environmental change. Current Opinion in Environmental Sustainability, 3(3), 142-149.

Sciubba, E. (2003). Extended exergy accounting applied to energy recovery from waste: The concept of total recycling. Energy, 28, 1315-1334.

Sempere, J. (2007). Los riesgos y el potencial político de la transición a la era post-petróleo. En: J. Sempere, \& E. Tello (Eds.), El final de la era del petróleo barato. Barcelona: Icaria editorial.

Suh, S. (2005). Theory of materials and energy flow analysis in ecology and economics. Ecological Modelling, 189, 251-269.

Sundkvist, Á., Jansson, A., Enefalk, Á. \& Larsson, P. (1999). Energy flow analysis as a tool for developing a sustainable society-a case study of a Swedish island. Resources, Conservation and Recycling, 25, 289299.

Talens, L., Villalba, G. \& Gabarrell, X. (2008). Exergy análisis of integrated waste Management in the recovery and recycling of used cooking oils. Environmental Science and Technology, 42 (13), 4977-4981. 
Thi Anh Tuyet, N. \& Ishihara, K., N. (2006). Analysis of changing hidden energy flow in Vietnam. Energy Policy, 34, 1883-1888.

Tratamiento y Selección de Residuos, S.A. (2008). TERSA: Solucions ambientals: Planta de Valorització Energètica (Sant Adrià de Besós): Dades de producció 2003-2007. Grupo TERSA. Extraído el 18 Enero, 2009 de www.tersa.com

Ulgiati, S., Odum, H.T. \& Bastianoni, S. (1994). Emergy use, environmental loading and sustainability. An emergy analysis of Italy. Ecological Modelling, 73, 215-268. 